Trauma Surgery \& Acute Care Open

\title{
Prehospital shock index outperforms hypotension alone in predicting significant injury in trauma patients
}

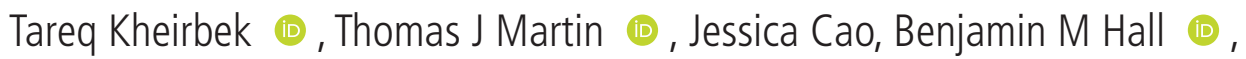 \\ Stephanie Lueckel (1), Charles A Adams
}

Department of Surgery, Brown University Warren Alpert Medical School, Providence, Rhode Island, USA

\section{Correspondence to} Dr Tareq Kheirbek; Tareq_ Kheirbek@brown.edu

The study is accepted for oral presentation at the 5th World Trauma Congress, February 2021. (c) Author(s) (or their employer(s)) 2021. Re-use permitted under CC BY-NC. No commercial re-use. See rights and permissions. Published by BMJ.

To cite: Kheirbek T, Martin TJ, Cao J, et al. Trauma Surg Acute Care Open 2021;6:e000712.

\section{ABSTRACT}

Background The American College of Surgeons Resources for Optimal Care of the Injured Patient recommends using hypotension, defined as systolic blood pressure $\leq 90 \mathrm{~mm} \mathrm{Hg}$, as an indicator of a full team trauma activation. We hypothesized that an elevated shock index (SI) predicts significant traumatic injuries better than hypotension alone.

Methods This is a retrospective cohort study analyzing full team trauma activations between February 2018 and January 2020, excluding transfers and those who had missing values for prehospital blood pressure or heart rate. We reviewed patients' demographics, prehospital and emergency department vitals, injury pattern, need for operation, and clinical outcomes. The primary outcome was rate of significant injury defined as identified injured liver, spleen, or kidney, pelvis fracture, long bone fracture, significant extremity soft tissue damage, hemothorax, or pneumothorax.

Results Among 544 patients, 82 (15.1\%) had prehospital hypotension and 492 had normal blood pressure. Of the patients with prehospital hypotension, $34(41.5 \%)$ had a significant injury. There was no difference in age, gender, medical history, or injury pattern between the two groups. There was no difference between the two groups in rate of serious injury $(41.5 \%$ vs. $46.1 \%$, NS), need for emergent operation (31.7\% vs. $28.1 \%$, NS) or death ( $20.7 \%$ vs. $18.8 \%$, NS). On the other hand, $\mathrm{SI} \geq 1$ was associated with increased rate of serious injury ( $54.6 \%$ vs. $43.4 \%$, $p=0.04)$. On a logistic regression analysis, prehospital hypotension was not associated with significant injury or need for emergent operation (OR $0.83,95 \% \mathrm{Cl} 0.51$ to 1.33 and OR 1.32, $95 \% \mathrm{Cl} 0.79$ to 2.25 , respectively). SI $\geq 1$ was associated with both increased odds of significant injury and need for emergent operation (OR $1.57,95 \% \mathrm{Cl} 1.01$ to 2.44 and $\mathrm{OR} 1.64,95 \% \mathrm{Cl} 1.01$ to 2.66$)$.

Discussion SI was a better indicator and could replace hypotension to better categorize and triage patients in need of higher level of care.

Level of evidence Prognostic and epidemiologic, level III.

\section{INTRODUCTION}

Appropriate triage is an essential element of the early resuscitation of the injured patient. Triage criteria of injured patients are well established and governed by the American College of Surgeons Committee on Trauma (ACS-COT). ${ }^{1}$ Current triage criteria are based on mechanism, physiology, and anatomic factors. Patients are typically triaged per tiered response system aimed at establishing a balance between optimizing patients' outcomes and the utilization of trauma system resources. Additionally, providers may activate the highest tier trauma response based on their discretion and dynamic clinical judgement. Hypotension remains the most clinically relevant factor to consider when triaging an injured patient and evaluating for a potential hemorrhagic injury. It is defined by the COT as the presence of systolic blood pressure (SBP) measurement of less than $90 \mathrm{~mm} \mathrm{Hg}$ at any time. Several studies have shown a correlation between hypotension and outcomes in trauma patients. Seamon et al notably demonstrated that even one episode of hypotension during resuscitation was significantly associated with a need for operative intervention. ${ }^{2}$ Others also showed that prehospital hypotension was associated with an increased injury severity, measured by Injury Severity Score (ISS), and need for transfusions. ${ }^{34}$

There are several conceptual and practical limitations to relying primarily on hypotension as the primary physiological metric for full trauma team activation. A cut-off of $90 \mathrm{~mm} \mathrm{Hg}$ has been chosen arbitrarily and blood pressure (BP) measurements are not the same for all age groups. ${ }^{5}$ The use of any static cut-off for a continuous measurement that varies across age groups will de facto place patients at risk for overtriage and undertriage. Patients with chronic medical conditions, such as cirrhosis, renal failure, or congestive heart failure, could present with a hypotension that is not related to injury. Similar presentation could be observed in those who are on antihypertensive medication regimens. Normotensive patients are not without a high risk of significant injury, either. ${ }^{6}$ Additionally, there is variability between automatic $\mathrm{BP}$ and manual $\mathrm{BP}$ measurement, as well as several human factors that result in possible inaccurate measurements. ${ }^{78}$ We anecdotally observed an overtriage of trauma patients at our institution as a result of using hypotension alone as an activation criterion. This, specifically, becomes a concern in trauma systems with limited resources.

Shock index (SI) has been proposed as an alternative metric for assessing the physiological response to hemorrhage. ${ }^{9}$ SI is calculated as the heart rate divided by SBP with values of 1 or above considered to be abnormal. SI accounts for the compensatory tachycardia seen in class II hemorrhagic shock 


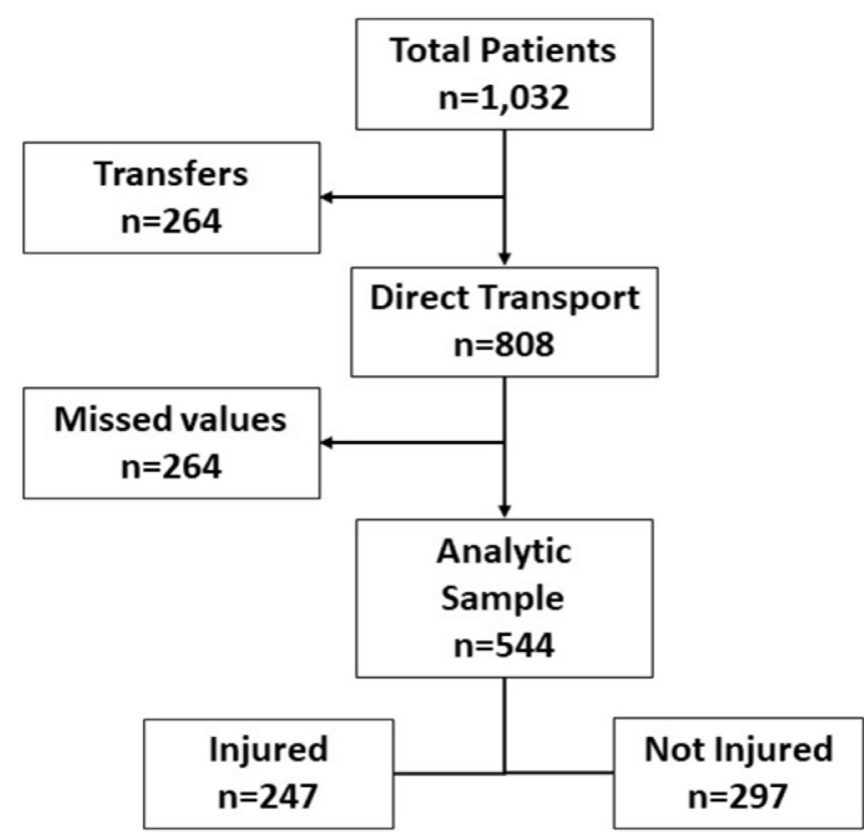

Figure 1 Flow diagram of selecting the analytic sample.

prior to the development of hypotension. King et al identified a cut-off of SI $>0.83$ as a marker for significant injury, ${ }^{10}$ whereas Mutschler et al proposed a redefinition of hypovolemic shock where SI $>1$ more accurately represented a class III shock. ${ }^{11} 12$

Table 1 Characteristics and outcomes of patients with and without prehospital hypotension

\begin{tabular}{|c|c|c|c|}
\hline & $\begin{array}{l}\text { Hypotensive } \\
(n=82)\end{array}$ & $\begin{array}{l}\text { Normotensive } \\
(n=462)\end{array}$ & $P$ value \\
\hline $\mathrm{SBP}$ (mm Hg, mean) & $80 \pm 9$ & $138 \pm 30$ & $<0.001$ \\
\hline Age (years, mean) & $51.7 \pm 18.8$ & $51.3 \pm 20.8$ & NS \\
\hline Gender (male) & $51(62.2 \%)$ & $326(70.6 \%)$ & NS \\
\hline Penetrating mechanism & $14(17.7 \%)$ & $62(13.4 \%)$ & NS \\
\hline Tourniquet use & $4(4.9 \%)$ & $7(1.5 \%)$ & NS \\
\hline \multicolumn{4}{|l|}{ Medical history } \\
\hline $\mathrm{CHF}$ & $1(1.2 \%)$ & $15(3.3 \%)$ & NS \\
\hline CKD/dialysis & $2(2.4 \%)$ & $3(0.7 \%)$ & NS \\
\hline Cirrhosis & $1(1.2 \%)$ & $7(1.5 \%)$ & NS \\
\hline Hypertension & $33(40.2 \%)$ & $163(35.3 \%)$ & NS \\
\hline Diabetes & $6(7.3 \%)$ & $52(11.3 \%)$ & NS \\
\hline Myocardial ischemia & $2(2.4 \%)$ & $6(1.3 \%)$ & NS \\
\hline Dementia & $2(2.4 \%)$ & $19(4.1 \%)$ & NS \\
\hline Alcohol abuse & $12(14.6 \%)$ & 75 (16.2\%) & NS \\
\hline Drug abuse & $12(14.6 \%)$ & 78 (16.9\%) & NS \\
\hline Steroid use & $1(1.2 \%)$ & $12(2.6 \%)$ & NS \\
\hline ED hypotension & $33(40.2 \%)$ & 70 (15.3\%) & 0.001 \\
\hline $\mathrm{SI}>1$ & $50(61 \%)$ & $47(10.1 \%)$ & 0.001 \\
\hline Significant injury & $34(41.5 \%)$ & $213(46.1 \%)$ & NS \\
\hline Transfusion (24 h) & $36(45.2 \%)$ & $158(38.9 \%)$ & NS \\
\hline Death in ED & $11(13.4 \%)$ & $33(7.1 \%)$ & NS \\
\hline Emergent operation* & $26(36.6 \%)$ & $130(30.3 \%)$ & NS \\
\hline In-hospital mortality* & $17(20.7 \%)$ & $87(18.8 \%)$ & NS \\
\hline ISS (median, IQR) & $13(5-22)$ & $17(9-26)$ & NS \\
\hline ISS $>16$ & $35(42.7 \%)$ & $245(53.2 \%)$ & NS \\
\hline
\end{tabular}

* Rates in patients who survived in the $E D$.

$\mathrm{CHF}$, congestive heart failure; CKD, chronic kidney disease; ED, emergency department; ISS, Injury Severity Score; ; NS, not significant; SBP, systolic blood pressure; SI, shock index.
We hypothesized that an elevated prehospital SI is better associated with significant traumatic injuries than prehospital hypotension alone; and therefore is a better indicator to trigger the full trauma team activation.

\section{METHODS}

This is a retrospective cohort study that aims to investigate association between prehospital hypotension or elevated SI with significant traumatic injuries, as a justification for the full trauma team activation at our level 1 trauma center. We reviewed our trauma registry to identify adult patients who were evaluated for full trauma team activation (level A) between February 2018 and January 2020. We excluded patients who were transferred from another institution, and those with missing values of prehospital BP or HR. After constructing the study concept, we obtained an Institutional Review Board approval prior to collecting the data, in accordance with the ethical standards laid down in the 1964 Declaration of Helsinki and its later amendments and in compliance of local state law.

We were interested in association of the physiological metrics with presence of a serious hemorrhagic injury. We identified this as a composite outcome of liver injury, splenic injury, pelvic fracture, long bone fracture, significant soft tissue injury that is associated with blood loss or needs operative intervention, hemothorax, or pneumothorax. The presence of at least one of these injuries was considered an occurrence of the outcome. Prehospital hypotension was defined as any SBP of $90 \mathrm{~mm} \mathrm{Hg}$ or less, as per COT guidelines and our hospital triage criteria. SI was calculated and patients were categorized as either having an SI $\geq 1$ or SI $<1$. Our secondary outcomes were: the need for emergent operation, the need for transfusion in the first 24 hours, death in the emergency department (ED) and in-hospital mortality. We also performed a stratified analysis to account for BP measurements in the ED.

Descriptive data are presented as frequencies for categorical variables, means for parametric continuous variables, and medians for non-parametric continuous variables. We applied Pearson's $\chi^{2}$ test with Fisher's exact test for sparse values to test independence for categorical data. Parametric continuous data were compared using Student's t-test. Non-parametric data were analyzed using Mann-Whitney test. Univariate logistic regression analyses were performed to obtain ORs of outcomes with hypotension or elevated SI. Since hypotension alone satisfies full trauma team activation per COT guidelines, we did not perform a multivariable logistic regression. Instead, stepwise logistic regression analyses were conducted to identify factors that are associated with our primary outcome, the need of transfusion, or ISS $\geq 16$. Receiver operating characteristic analysis was performed to assess predictability of the different predictors (hypotension, elevated SI, and the identified predicting models) with outcomes. Significance was set at $\alpha=0.05$. We completed all analyses using Stata/SE statistical software V.14.0 for Windows V.10, copyright 1985-2015 StataCorp, College Station, Texas, USA.

\section{RESULTS}

We identified 1032 patients who were evaluated at our ED for full trauma team-level trauma activation. After excluding transferred patients and those with missing values, our analytic sample was composed of 544 patients (figure 1). Of those, 82 had prehospital hypotension and 462 were normotensive, whereas 247 (45.4\%) had a significant injury, as defined by our composite outcome. 
Table 2 Outcomes of patients with and without prehospital elevated shock index (SI)

\begin{tabular}{llcc}
\hline & SI $>1(n=97)$ & SI $<1(\mathrm{n}=447)$ & P value \\
\hline Prehospital hypotension & $50(51.6 \%)$ & $32(7.2 \%)$ & $<0.001$ \\
Significant injury & $53(54.6 \%)$ & $194(43.4 \%)$ & 0.04 \\
Transfusion (24 h) & $60(63.8 \%)$ & $134(34.1 \%)$ & $<0.001$ \\
Death in ED & $13(13.4 \%)$ & $31(6.9 \%)$ & 0.04 \\
Emergent operation* & $34(35.1 \%)$ & $122(27.3 \%)$ & 0.04 \\
\hline In-hospital mortality* & $23(23.7 \%)$ & $81(18.1 \%)$ & NS
\end{tabular}

* Rates in patients who survived in the ED.

$E D$, emergency department; NS, not significant.

\section{Association of hypotension with significant injury}

We did not observe significant differences between those who had prehospital hypotension or were normotensive in demographics, mechanism, or medical history (table 1). Patients with prehospital hypotension were more likely to remain hypotensive in ED and more likely to have a prehospital SI $\geq 1$. However, there was no statistically significant association between prehospital hypotension and presence of a significant injury $(41.5 \%$ vs. $46.1 \%$, OR $0.83,95 \%$ CI 0.51 to 1.33 , NS), or need for transfusion $(45.2 \%$ vs. $38.9 \%$, NS). In those who survived the ED phase, prehospital hypotension was not associated with the need for emergent operation (OR 1.33, 95\% CI 0.79 to 2.25 ) or mortality (OR $0.64,95 \%$ CI 0.27 to 1.55 ). There was also no difference in ISS between the two groups. Using hypotension to trigger the full trauma team activation was associated with a high overtriage rate as evident with the rate of patients with ISS $<16(57.3 \%)$.

\section{Association of SI with significant injury}

Prehospital SI higher than 1 was associated with a higher rate of significant injury $(54.6 \%$ vs. $43.4 \%$, OR $1.57,95 \%$ CI 1.01 to 2.44, $\mathrm{p}=0.04)$, need for transfusion, death in ED, and need for emergent operation if the patient survived in ED (OR 1.64, 95\% CI 1.01 to 2.66) (table 2). There was no difference in in-hospital mortality (OR $0.99,95 \%$ CI 0.48 to 2.03 ).

We also performed a receiver operating characteristic analysis. Prehospital SI outperformed hypotension alone in predicting significant injury and need for transfusion. However, the difference was only significant for transfusion $(p=0.0001)$ (figure 2$)$.

\section{Stratified analysis}

We performed a stratified analysis to further identify presence of injury in a combination of scenarios. We found that elevated prehospital SI was specifically predictive of significant injury in those who were normotensive in the prehospital settings. In this population, the rate of significant injury was $61.7 \%$ in patients with elevated SI compared with $44.3 \%$ in those with an SI $<1$ $(p=0.03)$. Although the rate was also higher in the subgroup of patients with prehospital hypotension, it did not reach statistical significance (48\% vs. $31.3 \%$, NS). Similarly, hypotension in the ED was specifically associated with significant injury in the patients who had normal prehospital SBP (58.6\% vs. $40.7 \%$, $\mathrm{p}=0.02)$.

\section{Predictive models}

To explore which combinations of factors were most predictive of presence of our composite outcome of significant injuries, the need for transfusion, or having an overall severe injury (defined by ISS $\geq 16$ ), we performed stepwise logistic regression for each of these outcomes using patients' demographics, injury patterns, and physiological metrics. All three models showed elevated prehospital SI to be predictive of presence of injury, need for transfusion, and ISS $\geq 16$. On the other hand, prehospital hypotension was negatively associated with these outcomes (table 3 ). Each of the three models had a greater area under the curve compared with the use of a single metric alone-hypotension or elevated SI (figure 3).

\section{DISCUSSION}

Our results show that prehospital hypotension alone was not predictive of presence of a significant injury, need for transfusion, need for emergent operation, or mortality. In contrast, an elevated prehospital SI was associated with these outcomes, overall and specifically in patients who were normotensive in the prehospital settings. Our analysis also demonstrated that a single physiological metric was a poor predictor of injuries and meaningful clinical outcomes when compared with more complex models combining injury patterns, patient's demographics and physiological metrics.

A tiered response to injured patients achieves a balance between delivering optimal and timely care and reducing strain on the trauma center resources. The COT triage criteria were

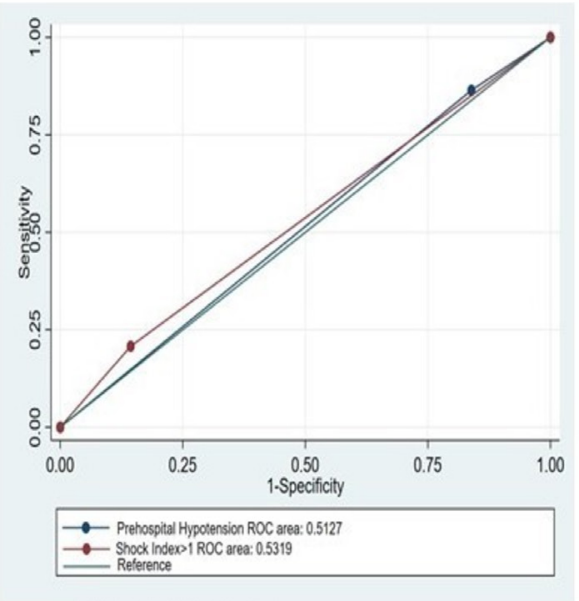

A Significant Injury

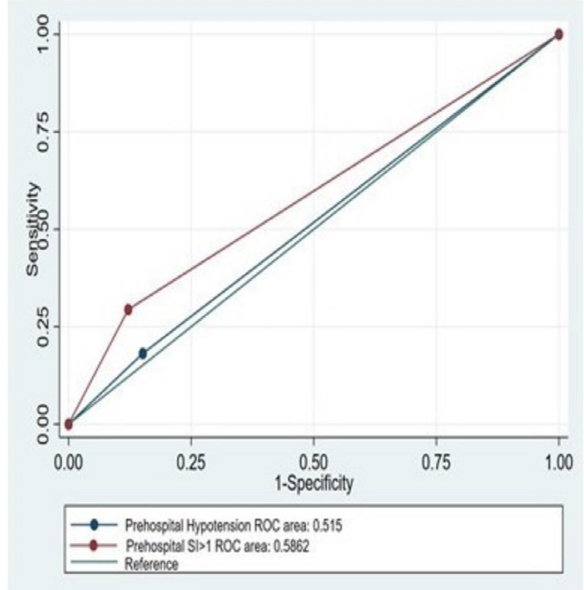

B Need for Transfusion, $\mathrm{p}=0.0001$

Figure 2 Receiver operating characteristic (ROC) curves comparing hypotension and elevated shock index (SI) for (A) presence of a significant injury and $(B)$ the need for transfusion. 
Table 3 Predictive models of significant injury, transfusion, and Injury Severity Score $\geq 16$ in our cohort based on the stepwise logistic regressions that were performed

\begin{tabular}{|c|c|c|}
\hline Models & $\mathrm{OR}$ & $95 \% \mathrm{Cl}$ \\
\hline \multicolumn{3}{|l|}{ Model for injury } \\
\hline Prehospital hypotension & 0.40 & 0.19 to 0.81 \\
\hline Prehospital SI >1 & 2.01 & 1.02 to 3.98 \\
\hline Age & 0.98 & 0.97 to 0.99 \\
\hline Penetrating trauma & 0.31 & 0.15 to 0.62 \\
\hline Alcohol use & 0.48 & 0.26 to 0.88 \\
\hline \multicolumn{3}{|l|}{ Model for transfusion } \\
\hline Prehospital hypotension & 0.28 & 0.13 to 0.63 \\
\hline Prehospital SI >1 & 5.14 & 2.49 to 10.60 \\
\hline Age & 1.01 & 1.01 to 1.03 \\
\hline ED hypotension & 3.84 & 2.14 to 6.89 \\
\hline \multicolumn{3}{|l|}{ Model for ISS $\geq 16$} \\
\hline Prehospital hypotension & 0.28 & 0.13 to 0.60 \\
\hline Prehospital SI >1 & 3.10 & 1.53 to 6.25 \\
\hline Male gender & 1.93 & 1.19 to 3.12 \\
\hline Penetrating trauma & 0.24 & 0.11 to 0.48 \\
\hline ED hypotension & 1.87 & 1.07 to 3.27 \\
\hline
\end{tabular}

ED, emergency department; ISS, Injury Severity Score; SI, shock index.

established to minimize overtriage without increasing undertriage, nor compromising the safety or outcomes of trauma patients. ${ }^{13}$ The criteria use injury mechanism, anatomic characteristics of injury, and physiological parameters to individually trigger different levels of trauma team activations. Hypotension, defined as SBP of $90 \mathrm{~mm} \mathrm{Hg}$ or below, has been used as the sole physiological parameter to prompt the full trauma team activation. ${ }^{1}$ Full trauma team activation adds more personnel to the response trauma team, as well as additional timely services provided by ancillary teams, such as respiratory therapies, radiology technologists, and so on. Unnecessary activation of full trauma team could result in disruption of the workflow of these teams in other hospital settings and adds a burden to healthcare systems with limited resources. Prehospital hypotension has a high sensitivity for suspecting a significant hemorrhagic injury, ${ }^{46}$ and several early studies showed an association with operative intervention and admission to an intensive care unit. ${ }^{314}$ However, it is possible that these findings are confounded by the widespread reliance of SBP $<90 \mathrm{~mm} \mathrm{Hg}$ as an indication for emergent intervention. Seamon et al suggested that a single episode of hypotension during resuscitation was associated with need for operative intervention and intensive care unit admission. ${ }^{2}$ However, our study adds to the other body of literature that challenges these associations.

There are several conceptual and practical limitations to relying primarily on hypotension as a physiological metric, especially when an isolated episode is considered adequate to initiate full trauma team activation. Hypotension alone is not always an accurate metric to predict outcome. ${ }^{15}$ Several authors have suggested that the arbitrary cut-off of $90 \mathrm{~mm} \mathrm{Hg}$ is not accurate, and possibly not sensitive enough to predict significant injury ${ }^{5}$ as normotensive patients are not without a high risk of significant injury, either. ${ }^{6}{ }^{16}$ Hypotension remains a single measurement in a complex disease process. There is a variability between automatic BP measurement and manual BP, as well as several human factors that result in possible inaccurate measurements. ${ }^{78}{ }^{17}$ In elderly patients with complex medical problems, hypotension is occasionally the cause of the hypotension episode on initial evaluation and simultaneously responsible for the injury, not the result of it. We anecdotally observed an overtriage of similar trauma patients at our institution as a result of using hypotension alone as an activation criterion. We noticeably encountered this in older patients with multiple comorbidities and complex preinjury polypharmacy regimens. Overtriage of patients with single episode of hypotension was also seen in patients with long prehospital transport time or transferred patients who were normotensive by time of arrival to our trauma center. When trauma team culture prioritizes protocol over critical analysis, we may encourage an overuse of unjustified therapy, and simultaneously disregard shock as it evolves before us. ${ }^{18}$

In our study, hypotension failed to predict presence of a significant injury or need for life-saving interventions. SI performed better than hypotension, being associated with $57 \%$ higher odds of significant injury and $64 \%$ higher odds of need for emergent operation. Interestingly, the association between SI and our primary outcome was especially strong in patients who were normotensive, which supports studies that have proposed raising the cut-off for hypotension to 105 or $110 \mathrm{~mm} \mathrm{Hg.}{ }^{2} 19$ This raises the concern that some 'normotensive' patients may not be appropriately triaged with full trauma team activation despite having significant injuries that otherwise could have been identified using the SI. We acknowledge that proposing a change in a long-standing definition would be practically challenging, specifically when extending such recommendations to a large number of seasoned trauma providers and prehospital emergency services personnel in a complex trauma system. However, our results support the use of SI as a supplementary measure to

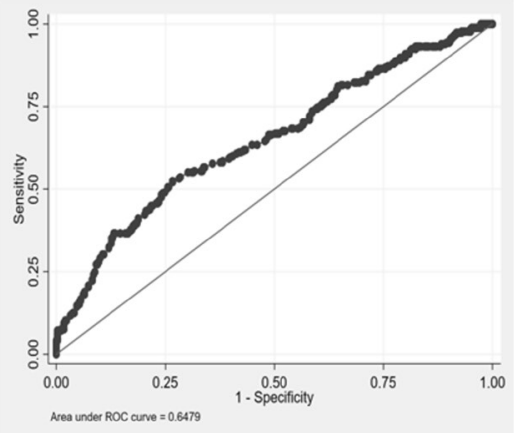

Model for significant injury

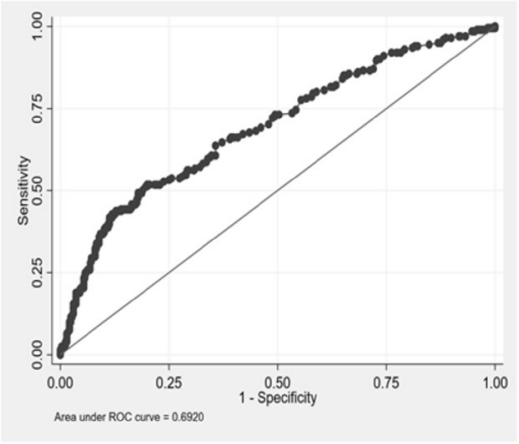

Model for need for transfusion

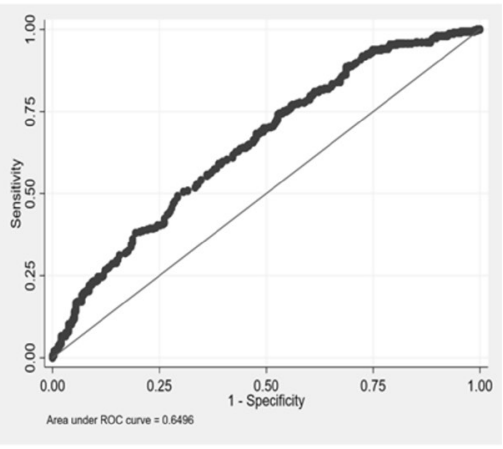

Model for $I S S \geq 16$

Figure 3 Receiver operating characteristic (ROC) curves for stepwise logistic regression models for significant injury, need for transfusion, and Injury Severity Score (ISS) $\geq 16$. 
accurately triage patients who are 'normotensive' despite other concerning findings related to anatomy or mechanism. ${ }^{2021}$ While the COT accounts for that with one criterion for full trauma team activation relying on provider's discretion, or 'gestalt', the SI serves as a way to numerate the provider's gestalt by adding a measured value to it. ${ }^{11} 1222$ The works of Vandromme et al and others support our findings in the normotensive trauma population..$^{21}$

The receiver operating characteristic analyses showed that an elevated SI outperformed prehospital hypotension. However, the area under the curve in either metric was still low. This emphasizes the complexity of trauma patients and the difficulty in constructing a universal predictive model in trauma, especially one that may be prospectively applied in the field at the time of injury. ${ }^{15}$ It also explains the plethora of scoring systems to predict injury severity and the need for transfusion ${ }^{23}$ where it appears that complex models with more variables perform better. ${ }^{24}{ }^{25}$ Our stepwise logistic regression shows that several factors combined predicted outcomes of clinical significance and injury severity. However, in all three models that we concluded, an elevated SI was consistently highly predictive, while prehospital hypotension was negatively associated with these outcomes.

One of the strengths of our study was that we used a composite outcome of clinically meaningful injuries that would validate the hypotension episode. Previous studies used the need for transfusion, the need for an operative intervention, or the admission to an intensive care unit as the primary outcome for their analyses. This does not address the potential provider's cognitive bias. If the importance of hypotension is overemphasized, providers are likely to make decisions which turn out to not be truly therapeutic. However, our study is not without limitations. This is a single-center study and results could be influenced by selection or information biases. We did not perform imputation for missed values, nor capture resuscitation efforts by our prehospital colleagues, both of which could add information bias to our analysis. Possible inaccurate BP measurements in the prehospital setting could represent another source of information bias. While we chose to study only patients who were evaluated as a level A activation, or full trauma team activation, as we were investigating the association of hypotension with appropriate triage per the COT guidelines, we think that could have added a selection bias that would be addressed by expanding it to other tier activations in the future.

\section{CONCLUSION}

In conclusion, though prehospital hypotension is an important criterion for full trauma team activation, our results suggest that elevated prehospital SI may outperform hypotension alone for the prediction of significant injury and need for emergent intervention. The ACS-COT should consider replacing hypotension with SI as the physiological metric to activate highest tier trauma response. Additionally, we suggest using elevated SI as an additional screening tool in patients who do not meet current ACS physiological criteria to decrease undertriage. It is more predictive of injury and can be calculated quickly by prehospital personnel and repeated by other trauma providers in the ED. An isolated physiological metric is not adequate as injury is often complex and multifactorial and a combination of different metrics, including physiological, anatomic, demographic metrics, in addition to mechanism is probably needed. This could be close to reality with recent advances in artificial intelligence and portable technologies. ${ }^{26}$ This has major implications on best utilization of regional trauma systems and hospitals, as well as when generalizing recommendations to other countries and regions with variable resources, where individualized criteria are likely to have a stronger impact. Future prospective investigations, such as pretriage and post-triage protocol adjustments, as well as a cost-effectiveness analysis of such adjustment should address some of the limitations in our study and provide further insight into the full benefits of modifying trauma triage criteria.

Contributors TK: conceptual design, data analyses, interpretation of results, article preparation, critical revisions. TJM: data analyses, interpretation of results, article preparation, critical revisions. JC, SL, BMH, CAA: interpretation of results, article preparation, critical revisions.

Funding The authors have not declared a specific grant for this research from any funding agency in the public, commercial or not-for-profit sectors.

Competing interests None declared.

Patient consent for publication Not required.

Ethics approval The local Institutional Review Board (IRB) reviewed and approved the study (approval number: 1596334-1). The IRB waived the informed consent requirement for this study due to the retrospective nature of data collection.

Provenance and peer review Not commissioned; externally peer reviewed.

Data availability statement Data may be obtained from a third party and are not publicly available

Open access This is an open access article distributed in accordance with the Creative Commons Attribution Non Commercial (CC BY-NC 4.0) license, which permits others to distribute, remix, adapt, build upon this work non-commercially, and license their derivative works on different terms, provided the original work is properly cited, appropriate credit is given, any changes made indicated, and the use is non-commercial. See: http://creativecommons.org/licenses/by-nc/4.0/.

\section{ORCID iDs}

Tareq Kheirbek http://orcid.org/0000-0002-6758-9150

Thomas J Martin http://orcid.org/0000-0002-6194-2830

Benjamin M Hall http://orcid.org/0000-0002-1324-7729

Stephanie Lueckel http://orcid.org/0000-0002-4393-3076

\section{REFERENCES}

1 American College of Surgeons CoT. Resources for optimal care of the injured patient, 2014.

2 Seamon MJ, Feather C, Smith BP, Kulp H, Gaughan JP, Goldberg AJ. Just one drop: the significance of a single hypotensive blood pressure reading during trauma resuscitations. J Trauma 2010;68:1289-95.

3 Lipsky AM, Gausche-Hill M, Henneman PL, Loffredo AJ, Eckhardt PB, Cryer HG, de Virgilio C, Klein SL, Bongard FS, Lewis RJ. Prehospital hypotension is a predictor of the need for an emergent, therapeutic operation in trauma patients with normal systolic blood pressure in the emergency department. J Trauma 2006:61:1228-33.

4 Damme CD, Luo J, Buesing KL. Isolated prehospital hypotension correlates with injury severity and outcomes in patients with trauma. Trauma Surg Acute Care Open 2016;1:e000013.

5 Bruns B, Gentilello L, Elliott A, Shafi S. Prehospital hypotension redefined. J Trauma 2008:65:1217-21.

6 Holcomb JB, Niles SE, Miller CC, Hinds D, Duke JH, Moore FA. Prehospital physiologic data and lifesaving interventions in trauma patients. Mil Med 2005;170:7-13.

7 Davis JW, Davis IC, Bennink LD, Bilello JF, Kaups KL, Parks SN. Are automated blood pressure measurements accurate in trauma patients? J Trauma 2003;55:860-3.

8 Skirton H, Chamberlain W, Lawson C, Ryan H, Young E. A systematic review of variability and reliability of manual and automated blood pressure readings. J Clin Nurs 2011;20:602-14.

9 Vandromme MJ, Griffin RL, Kerby JD, Rue LW, Rue LW, Weinberg JA. Identifying risk for massive transfusion in the relatively normotensive patient: utility of the prehospital shock index. J Trauma 2011;70:384-90.

10 King RW, Plewa MC, Buderer NM, Knotts FB. Shock index as a marker for significant injury in trauma patients. Acad Emerg Med 1996;3:1041-5.

11 Mutschler M, Nienaber U, Münzberg M, Wölfl C, Schoechl H, Paffrath T, Bouillon B, Maegele $M$. The shock index revisited - a fast guide to transfusion requirement? A retrospective analysis on 21,853 patients derived from the TraumaRegister DGU. Crit Care 2013;17:R172.

12 Fröhlich M, Driessen A, Böhmer A, Nienaber U, Igressa A, Probst C, Bouillon B, Maegele $M$, Mutschler $M$. Is the shock index based classification of hypovolemic shock applicable in multiple injured patients with severe traumatic brain injury?an analysis of the TraumaRegister DGU®. Scand J Trauma Resusc Emerg Med 2016;24:148. 
13 Cook CH, Muscarella P, Praba AC, Melvin WS, Martin LC. Reducing overtriage without compromising outcomes in trauma patients. Arch Surg 2001;136:752-6.

14 Shapiro NI, Kociszewski C, Harrison T, Chang Y, Wedel SK, Thomas SH. Isolated prehospital hypotension after traumatic injuries: a predictor of mortality? J Emerg Med 2003;25:175-9.

15 Liu NT, Holcomb JB, Wade CE, Salinas J. Inefficacy of standard vital signs for predicting mortality and the need for prehospital life-saving interventions in blunt trauma patients transported via helicopter: a repeated call for new measures. J Trauma Acute Care Surg 2017;83:S98-103.

16 Edelman DA, White MT, Tyburski JG, Wilson RF. Post-traumatic hypotension: should systolic blood pressure of 90-109 mmHg be included? Shock 2007;27:134-8.

17 Prasad NH, Brown LH, Ausband SC, Cooper-Spruill O, Carroll RG, Whitley TW. Prehospital blood pressures: inaccuracies caused by ambulance noise? Am J Emerg Med 1994;12:617-20

18 Kheirbek T, Jikaria N, Murray B, Martin TJ, Lueckel SN, Stephen AH, Monaghan SF, Adams CA. Unjustified administration in liberal use of tranexamic acid in trauma resuscitation. J Surg Res 2021;258:125-31.

19 Eastridge BJ, Salinas J, McManus JG, Blackburn L, Bugler EM, Cooke WH, Convertino VA, Concertino VA, Wade CE, Holcomb JB. Hypotension begins at $110 \mathrm{~mm} \mathrm{Hg}$ : redefining "hypotension" with data. J Trauma 2007;63:291-9.

20 Cannon CM, Braxton CC, Kling-Smith M, Mahnken JD, Carlton E, Moncure M. Utility of the shock index in predicting mortality in traumatically injured patients. J Trauma 2009;67:1426-30
21 Singh A, Ali S, Agarwal A, Srivastava RN. Correlation of shock index and modified shock index with the outcome of adult trauma patients: a prospective study of 9860 patients. N Am J Med Sci 2014;6:450-2.

22 Pottecher J, Ageron F-X, Fauché C, Chemla D, Noll E, Duranteau J, Chapiteau L, Payen $J-F$, Bouzat P. Prehospital shock index and pulse pressure/heart rate ratio to predict massive transfusion after severe trauma: retrospective analysis of a large regional trauma database. J Trauma Acute Care Surg 2016;81:713-22.

23 Callcut RA, Cotton BA, Muskat P, Fox EE, Wade CE, Holcomb JB, Schreiber MA, Rahbar $\mathrm{MH}$, Cohen MJ, Knudson MM, et al. Defining when to initiate massive transfusion: a validation study of individual massive transfusion triggers in PROMMTT patients. $J$ Trauma Acute Care Surg 2013;74:59-65.

24 Brockamp T, Nienaber U, Mutschler M, Wafaisade A, Peiniger S, Lefering R, Bouillon $B$, Maegele M. Predicting on-going hemorrhage and transfusion requirement after severe trauma: a validation of six scoring systems and algorithms on the TraumaRegister DGU®. Crit Care 2012;16:R129.

25 McNab A, Burns B, Bhullar I, Chesire D, Kerwin A. An analysis of shock index as a correlate for outcomes in trauma by age group. Surgery 2013;154:384-7.

26 Kang D-Y, Cho K-J, Kwon O, Kwon J-M, Jeon K-H, Park H, Lee Y, Park J, Oh B-H. Artificial intelligence algorithm to predict the need for critical care in prehospital emergency medical services. Scand J Trauma Resusc Emerg Med 2020;28:17. 